\title{
Antithyroid drugs used in the treatment of hyperthyroidism during breast feeding. An update and new perspectives
}

\author{
Spyros Karras, Themistoklis Tzotzas, Gerasimos E. Krassas
}

Department of Endocrinology, Diabetes and Metabolism, Panagia General Hospital, Thessaloniki, Greece

Antithyroid drugs (ATD) are widely used by endocrinologists all over the world for the treatment of Graves' disease (GD) in the general population and in lactating thyrotoxic mothers. ${ }^{1,2}$ Traditionally, these agents produce minor side effects (rash, fever, urticaria) in $5-10 \%$ of the treated patients and major side effects (agranulocytosis, vasculitis, hepatic toxicity) much less frequently. These side effects appear more likely to be dose-related for methimazole (MMI) rather than propylthiouracil (PTU). ${ }^{3,4}$ To our knowledge, there are no specific data regarding the occurrence of minor side effects that might occur in lactating thyrotoxic mothers under ATD. Nevertheless, daily clinical practice has usually shown these side effects to be reversible after dose adjustment.

Both MMI and PTU appear to be effective for the treatment of hyperthyroidism. It seems, however, that MMI has certain advantages over PTU such as better adherence due to its once daily regimen and fewer major side effects (vasculitis, hepatotoxicity). ${ }^{3-5}$

Key words: Antithyroid drugs, Breast feeding, Graves' disease, Lactating thyrotoxic mothers, Methimazole, Propilthiuracil

Address for correspondence:

Gerasimos E. Krassas, MD, PhD, FRCP (Lond), Chairman, Department of Endocrinology, Diabetes and Metabolism, Panagia General Hospital, N. Plastira 22, N. Krini 55132, Thessaloniki, Greece, Tel. +302310479633,

Fax: +302310282476, e-mail: krassas@the.forthnet.gr

Received 28-08-09, Revised 15-09-09, Accepted 20-09-09
Additionally, both MMI and PTU elicit multiple immunosuppressive effects in hyperthyroid patients by inducing apoptosis of intrathyroidal leucocytes and decreasing the number of $\mathrm{T}$ helper cells and NK cells. ${ }^{6,7}$ In a recent study, ${ }^{8} 30 \%$ of patients who had previously been treated with ATD and were no longer on treatment (for 1-56 weeks) had positive antineutrophil cytoplasmic antibodies, especially when PTU was the drug of choice. Other studies have also confirmed these results. ${ }^{9,10}$

Although important data have been accumulated from many elegant studies conducted over the last two decades regarding ATD and their compatibility with lactation, ${ }^{11-16}$ special attention must be paid to new information regarding their harmful effects on liver function. The issue of PTU use in the adult and pediatric thyrotoxic population has emerged during the last year. Since $1946,{ }^{17}$ a good number of reports on severe hepatic dysfunction have been published regarding both ATD, and especially as concerns PTU, in children and adults with hyperthyroidism. ${ }^{18-20}$

A cohort study in $1993^{21}$ evaluated the incidence, severity and course of hepatic dysfunction in 54 hyperthyroid patients treated with PTU who had normal liver enzymes before treatment. They found that 15 patients presented alanine transferase (ALT) elevations after two months of treatment, which reversed to normal in 13 of them after dose reduction. In the same study the authors found approximately 20 published cases of PTU liver toxicity. In 2001, Kim et $\mathrm{al}^{22}$ also evaluated the incidence of PTU-liver toxicity in 497 adult hyperthyroid patients, without 
previous abnormal liver tests, which, as is well known, occurs in up to $25-30 \%$ of thyrotoxic patients. Six patients $(1.2 \%)$ developed hepatic dysfunction after 12-49 days of treatment. Abnormal liver function tests normalized in all cases after 16-145 (72 \pm 46.4$)$ days of PTU withdrawal. The authors concluded that most symptomatic hepatic injury developed during the first months of therapy and had a benign course after drug discontinuation. They also proposed the monitoring of liver function tests during PTU administration. Lian et $\mathrm{al}^{23}$ in 2004 presented similar results, although they concluded that it is difficult to predict the injury development. They also suggested monitoring of the liver function during the first months of PTU treatment.

In 2005, Cooper ${ }^{4}$ suggested that PTU should be considered as a second line agent for thyrotoxicosis treatment and recommended that it should be used only in particular clinical situations such as thyroid storm, allergic reactions to previous MMI treatment (although there is a 50\% cross reactivity between the two drugs) and in the first trimester of pregnancy because of the possible teratogenic effects of MMI. ${ }^{24,25}$ At present, both drugs are considered safe during lactation. ${ }^{2}$ For several reasons, MMI is not available in some countries and PTU is still used as a first line treatment.

In October $2008,{ }^{26}$ a meeting was held sponsored by the Eunice Kennedy Shriver Institute of Health and Human Development to reevaluate PTU safety for pediatric Graves' disease and, on this occasion, examined the recent data regarding PTU-liver toxicity in children. An extensive review of the data showed that there were in total 33 published case reports of PTU-liver failure in adults and 14 in children. There was a great time variation in the occurrence of liver failure (6-450 days, median 120 days). Of major concern, 27 liver transplants (16 in adults and 7 in children) and nine deaths in adults and three deaths in children have been reported in the United States following PTU use during the last 18 years. Based on these data, experts in the field strongly recommended that prescription of PTU in the pediatric population be ceased.

A second meeting sponsored by the American Thyroid Association (ATA) and the Food and Drug
Administration (FDA) of the United States was held in April $2009{ }^{27}$ which reevaluated, among others, the problem of PTU-liver toxicity in children and adults. Based on the existing data on the prevalence of Graves' thyrotoxicosis and PTU related severe liver damage among the US population, it was estimated that 3-4 adults or children per year will develop severe liver failure necessitating immediate transplantation. Although pregnancy data are not available, estimating a $0.1 \%$ frequency of autoimmune hyperthyroidism during pregnancy, it was calculated that four pregnant women per year will develop severe hepatic failure using PTU in the US. However, to the best of our knowledge, there have been no case reports of liver failure (or transplant) in women with GD after or during pregnancy, at least in Europe.

It has been proven that most of these cases were idiosyncratic, non-dose-related and presented a latent period of occurrence. There were no biomarkers that could predict hepatic damage, making routine monitoring of liver function ineffective in terms of cost of screening, contrasting with what has been previously published on PTU-liver toxicity.

Recently, the Hyperthyroidism Guidelines Task Force of the ATA ${ }^{28}$ recommended that PTU should not be prescribed as the first line agent in children or adults. Its use might be accepted in the first trimester of pregnancy, in severe thyrotoxicosis or thyroid storm or in patients exhibiting adverse reactions to previous MMI treatment. The risk of developing severe hepatic failure remains unacceptable despite its rarity in all population groups. These recommendations do not consider routine follow-up of liver function as a useful tool in preventing or diagnosing PTU-liver damage. The best way to prevent these dramatic clinical conditions is not to prescribe PTU except in the settings mentioned above.

In these recommendations there are no particular comments concerning the possible use of PTU in lactating mothers. If we consider that a significant percentage of these women wish to breastfeed and the fact that they commonly experience a flare of autoimmune hyperthyroidism after pregnancy, the question of treatment remains unsolved. Even if we assume that the risk for nursing infants under PTU is minimal, because of the inconsequential amount 
of the excreted drug into human milk, ${ }^{29,30}$ we cannot safely exclude a risk for hepatic injury in the lactating mothers. Moreover, the issue of the amount of excreted drug in the milk and the possible risks for the infant cannot be disregarded. These adverse effects, especially as regards PTU, are not doserelated, which means that even a small amount of drug could potentially be harmful for the infant and hepatotoxicity may appear after a latent period ranging from several months to several years. To the best of our knowledge, the studies with the longest surveillance time were conducted up to the age of 6 years for these children ${ }^{15,16}$ and the parameters which have been assessed were thyroid status and somatic and intellectual development and not their immunologic profile up to several years. A significant percentage of these women are at potential risk for developing PTU toxicity, as the disease often flares up postpartum and larger doses of these drugs are needed during this period.

Based on the current guidelines ${ }^{2,31}$ on the general use of ATD - and PTU in particular-several questions are emerging concerning the particular group of lactating mothers. Should these women be left on PTU treatment, which is minimally excreted in human milk, or should we switch them to MMI therapy? Is routine follow-up advisable in this case, bearing in mind that although much less common, MMI could be hepatotoxic as well? Of particular concern are small for gestational age infants or those with impaired vital functions (hepatic, renal). Additionally, should we adjust the dose of MMI to the minimal effective one in the mothers of these children? Should we continuously treat mothers with PTU/MMI or should we suggest thyroidectomy after euthyroidism is achieved, since RAI is contraindicated? Which would be the optimal period of ATD treatment for this? All these concerns need to be addressed in this specific population, i.e. the lactating mothers and their infants.

At present, it is recommended that PTU treatment be avoided for thyrotoxicosis in lactating mothers as it is suggested in the general adult and pediatric population. MMI use is still recommended under surveillance, especially in some cases (small infants, mothers with health problems) at the minimal doses that can lead to euthyroidism. Alternatively, surgical management could be a rational option until longer and larger studies confirm the long-term safety of MMI. In our opinion, the appropriate management for these women has to balance the risks and benefits on this issue. The goal is to maintain lactation without compromising the mothers' hepatic function and the infants' immunological profile. Other therapeutic alternatives, e.g. surgery, could be considered during this period of time.

Finally, the authors of this report propose that international and national thyroid societies (such as ETA, ATA, etc.) invite their members to review all retrospective data available concerning long-term liver function in women who breastfed and were treated by ATD during the postpartum period. Since the number of such cases is limited, such data could probably be retrieved from hospital records and, no doubt, will help to provide the appropriate guidelines to all members with regard this very important issue.

In conclusion, in the absence of adequate data regarding the impact of ATD treatment on lactating hyperthyroid women and considering the recent data of the potential harmful effects of PTU, MMI should be used instead, at the minimal doses, in that specific situation. Further studies are needed before we reach final conclusions on these important issues.

\section{REFERENCES}

1. Mandel SJ, Cooper DS, 2001 The use of antithyroid drugs in pregnancy and lactation. J Clin Endocrinol Metab 86: 2354-2359.

2. Abalovich M, Amino N, Barbour LA, et al, 2007 Management of thyroid dysfunction during Pregnancy and Postpartum. J Clin Endocrinol Metab 92: Suppl 8: S1S47.

3. Cooper DS, 2003 Antithyroid drugs in the management of patients with Graves' disease: an evidence-based approach to therapeutic controversies. J Clin Endocrinol Metab 88: 3474-3481.

4. Cooper DS, 2005 Antithyroid drugs. N Engl J Med 352: 905-917.

5. He CT, Hsieh AT, Pei D, et al, 2004 Comparison of single daily dose of methimazole and propylthiouracil in the treatment of Graves' hyperthyroidism. Clin Endocrinol (Oxf) 60: 676-681.

6. Tsatsoulis A, Vlachoyiannopoulos PG, Dalekos GN, Johnson EO, Moutsopoulos HM, 1995 Increased serum interleukin-1 beta during treatment of hyperthyroidism 
with antithyroid drugs. Eur J Clin Invest 25: 654-658.

7. Mitsiades N, Poulaki V, Tseleni-Balafouta S, Chrousos GP, Koutras DA, 2000 Fas ligand expression in thyroid follicular cells from patients with thionamide-treated Graves' disease. Thyroid 10: 527-532.

8. Harper L, Chin L, Daykin J, et al, 2004 Propylthiouracil and carbimazole associated-antineutrophil cytoplasmic antibodies (ANCA) in patients with Graves' disease. Clin Endocrinol (Oxf) 60: 671-675.

9. Gao Y, Chen M, Ye H, Yu F, Guo XH, Zhao MH, 2008 Long-term outcomes of patients with propylthiouracilinduced anti-neutrophil cytoplasmic auto-antibody-associated vasculitis. Rheumatology (Oxf) 47: 1515-1520.

10. Sato H, Hattori M, Fujieda M, et al, 2000 High prevalence of antineutrophil cytoplasmic antibody positivity in childhood onset Graves' disease treated with propylthiouracil. J Clin Endocrinol Metab 85: 4270-4273.

11. Azizi F, 1996 Effect of methimazole treatment of maternal thyrotoxicosis on thyroid function in breast-feeding infants. J Pediatr 128: 855-858.

12. Azizi F, Hedayati M, 2002 Thyroid function in breast-fed infants whose mothers take high doses of methimazole. J Endocrinol Invest 25: 493-496.

13. Azizi F, 2003 Thyroid function in breast-fed infants is not affected by methimazole-induced maternal hypothyroidism: results of a retrospective study. J Endocrinol Invest 26: 301-304.

14. Ordookhani A, Pearce EN, Hedayati M, et al, 2007 Assessment of thyroid function and urinary and breast milk iodine concentrations in healthy newborns and their mothers in Tehran. Clin Endocrinol (Oxf) 67: 175-179.

15. Azizi F, Khoshniat M, Bahrainian M, Hedayati M, 2000 Thyroid function and intellectual development of infants nursed by mothers taking methimazole. J Clin Endocrinol Metab 85: 3233-3238.

16. Azizi F, Bahrainian M, Khamseh ME, Khoshniat M, 2003 Intellectual development and thyroid function in children who were breast-fed by thyrotoxic mothers taking methimazole. Pediatr Endocrinol Metab 16: 1239-1243.

17. Moore FD, 1946 Toxic manifestations of thiouracil therapy. JAMA 130: 315-319.

18. Arab DM, Malatjalian DA, Rittmaster RS, 1995 Severe cholestatic jaundice in uncomplicated hyperthyroidism treated with methimazole. J Clin Endocrinol Metab 80: 1083-1085.

19. Williams KV, Nayak S, Becker D, Reyes J, Burmeister LA, 1997 Fifty years of experience with propylthiouracil- associated hepatotoxicity: what have we learned? J Clin Endocrinol Metab 82: 1727-1733.

20. Ruiz JK, Rossi GV, Vallejos HA, Brenet RW, Lopez IB, Escribano AA, 2003 Fulminant hepatic failure associated with propylthiouracil. Ann Pharmacother 37: 224-228.

21. Liaw YF, Huang MJ, Fan KD, Li KL, Wu SS, Chen TJ, 1993 Hepatic injury during propylthiouracil therapy in patients with hyperthyroidism. A cohort study. Ann Intern Med 118: 424-428.

22. Kim HJ, Kim BH, Han YS, et al, 2001 The incidence and clinical characteristics of symptomatic propylthiouracilinduced hepatic injury in patients with hyperthyroidism: a single-center retrospective study. Am J Gastroenterol 96: 165-169.

23. Lian XL, Bai Y, Dai WX, Jin ZM, Zeng ZP, Guo ZS, 2004 The clinical characteristics of symptomatic propylthiouracil-induced hepatic injury in patients with hyperthyroidism. Zhonghua Nei Ke Za Zhi 43: 442446.

24. Van Dijke CP, Heydendael RJ, De Kleine MJ, 1987 Methimazole, carbimazole, and congenital skin defects. Ann Intern Med 106: 60-61.

25. Barbero P, Valdez R, Rodríguez H, et al, 2008 Choanal atresia associated with maternal hyperthyroidism treated with methimazole: a case-control study. Am J Med Genet A 146A: 2390-2395.

26. Eunice Kennedy Shriver National Institute of Child Health and Human Development, 2008 Hepatic toxicity following treatment for pediatric Graves' disease. Minutes of October 282008 meeting.

27. U.S. Food and Drug Administration, 2009 Propylthiouracyl (PTU)-related liver toxicity. April 182009 workshop.

28. Bahn RS, Burch HS, Cooper DS, et al, 2009 The Role of Propylthiouracil in the Management of Graves' Disease in Adults: report of a meeting jointly sponsored by the American Thyroid Association and the Food and Drug Administration. Thyroid 19: 673-674.

29. Kampmann JP, Johansen K, Hansen JM, Helweg J, 1980 Propylthiouracil in human milk. Revision of a dogma. Lancet 1: 736-737.

30. Low LC, Lang J, Alexander WD, 1979 Excretion of carbimazole and propylthiouracil in breast milk. Lancet 2: 1011.

31. American Academy of Pediatrics Committee on Drugs, 2001 Transfer of drugs and other chemicals into human milk. Pediatrics 108: 776-789. 